\title{
ATLAS SemiConductor Tracker: Operation and Performance
}

\author{
M. Moreno Llácer, for the ATLAS SCT collaboration \\ Instituto de Física Corpuscular (IFIC), CSIC-UV \\ P.O. Box 22085, E-46071, Valencia, Spain \\ E-mail: mamolla@cern.ch
}

\begin{abstract}
The SemiConductor Tracker (SCT) is a silicon strip detector and one of the key precision tracking devices in the Inner Detector of the ATLAS (A Toroidal LHC ApparatuS) experiment at CERN. The SCT installation in the ATLAS experimental cavern was completed in 2007 and it has been operational since then. An extensive commissioning phase followed, during which calibration data was collected and the performance of the system was studied. The SCT was ready for the first LHC proton-proton collisions and it continues recording data successfully since then. In this paper, the current status, operation and performance of the SCT is reviewed, including results from data-taking periods in 2010 and 2011.
\end{abstract}

Index Terms-Silicon microstrips, ATLAS, noise, radiation damage.

\section{INTRODUCTION}

A TLAS (A Toroidal LHC ApparatuS) [1] is one of the two large general purpose detectors built at the Large Hadron Collider (LHC) [2] to explore physics at $\mathrm{TeV}$ scale. It consists of several detector subsystems. Closest to the interaction point is the Inner Detector [3]. This charged particle tracker is immersed in a $2 T$ axial magnetic field provided by a superconducting solenoidal magnet. The Inner Detector consists of three subsystems: the Pixel Detector, the SemiConductor Tracker (SCT) and the Transition Radiation Tracker (see Fig. 1). The first two are silicon-based whereas the latter one is a gaseous detector.

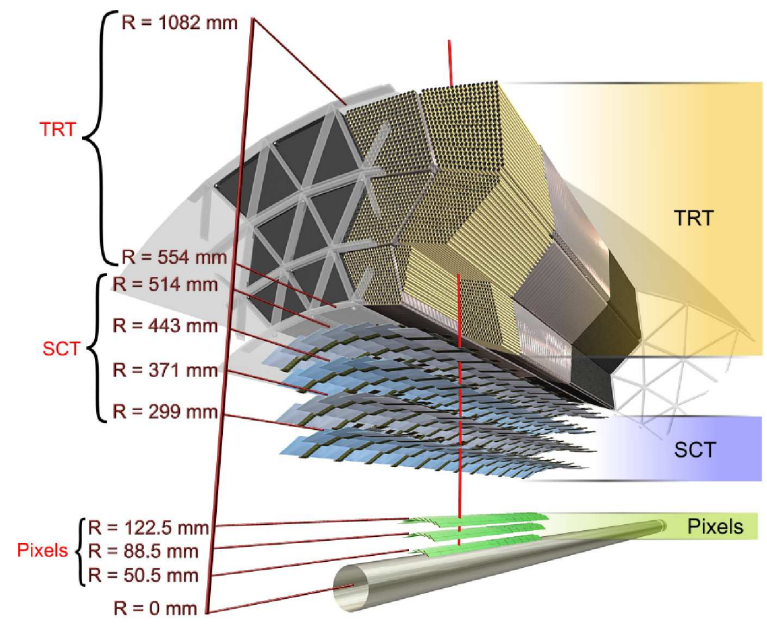

Fig. 1. A schematic view of the ATLAS Inner Detector.
ATLAS started proton-proton collision data taking in December 2009. The integrated luminosity recorded in 2009 during stable beams and for collisions at $900 \mathrm{GeV}$ centre-of-mass energy is $12 \mu b^{-1}$. The LHC collision energy was increased to $7 \mathrm{TeV}$ in March 2010 and ATLAS recorded $45 \mathrm{pb}^{-1}$ of $7 \mathrm{TeV}$ proton-proton collision data in 2010 and more than $5 \mathrm{fb}^{-1}$ in 2011.

\section{The ATLAS SEmiConductor Tracker}

With $61 \mathrm{~m}^{2}$ of active silicon area and 6.3 million readout channels, the SCT provides precision measurements of charged particle tracks in the radial range from 30 to $52 \mathrm{~cm}$. The whole SCT includes 4088 silicon micro-strip modules and occupies a cylinder volume of $5.6 \mathrm{~m}$ in length and $52 \mathrm{~cm}$ in radius. The detector is subdivided into barrel and endcap regions, as illustrated in Fig. 2. The barrel region consists of four layers and covers the pseudorapidity range $|\eta|<1$. Each endcap consists of nine disks and extends the coverage to $|\eta|<2.5$.

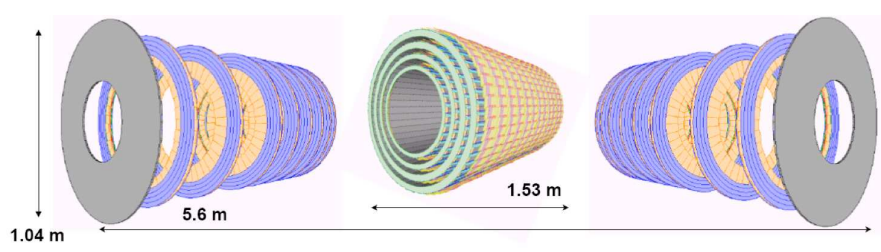

Fig. 2. SCT geometry: barrel layers in the center and one endcap in each side.

The SCT was designed to fulfill the following requirements:

- Intrinsic strip efficiency $>99 \%$.

- Noise occupancy $<5 \times 10^{-4}$.

- $1 \%$ maximum of non-operational strips.

- Robust pattern recognition.

- Excellent momentum resolution and vertex reconstruction.

The elementary structural unit of the detector are the readout modules (Fig. 3), amounting a total of 2112 in the barrel and 968 in each endcap. All barrel modules are of the same type [4], whereas there are four types in the endcap that mainly differ in shape [5]. Each module consists of two 
pairs of single-sided 'p-in-n' microstrip silicon sensors (4 sensors/module) glued back-to-back at a stereo angle of $40 \mathrm{mrad}$ to provide a two dimensional track reconstruction. Each sensor features 768 strips and its thickness is $285 \mu \mathrm{m}$. The pitch is $80 \mu \mathrm{m}$ for the barrel modules (aligned parallel to the solenoidal field) and varies from 57 to $94 \mu \mathrm{m}$ for the endcap modules (aligned radially). This strip pitch gives a single-hit resolution of $\sim 16 \mu \mathrm{m}$ in the bending plane.

The readout of each module is based on 12 ABCD3TA ASICs [6] manufactured in the radiation-hard DMILL technology. They are mounted on a copper/kapton hybrid next to the sensor edge. Each chip reads out 128 strips. It has a programmable charge threshold and provides binary hit information (i.e. 'hit' or 'no hit').

The communication between the SCT modules and the offdetector electronics is handled by optical fibre connections [7]. Clock and command signals are sent from the crates to the modules through ' $\mathrm{TX}$ ' fibres (one fibre per module) while data is returned to the crates through ' $R X$ ' fibres (one per module side). Both ' $\mathrm{TX}$ ' and ' $\mathrm{RX}$ ' links have a redundancy scheme in case of fibre breaks or transmitter failures. For the ' $\mathrm{TX}$ ', it is possible for a module to receive clock and command signals electronically from a neighbouring module. For the ' $R X$ ', it is possible to read out both sides of the module through one of the two ' $\mathrm{RX}$ ' links. The detector control and data handling is performed off-detector by the readout driver board [8].
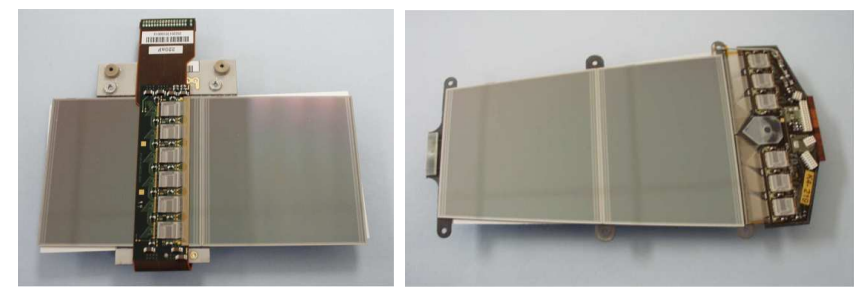

Fig. 3. Pictures of the SCT barrel (left) and endcap (right) readout modules.

To limit the radiation damage and to decrease the noise levels, the SCT detector is cooled down by an evaporative cooling system which uses $\mathrm{C}_{3} \mathrm{~F}_{8}$ [9]. The cooling circuits are in direct contact with each of the SCT modules. The modules baseboard serves as the thermal conductor to remove the heat generated by the readout electronics and sensors.

\section{Operation OF THE SEMICONDUCTOR TRACKER}

The SCT installation in the ATLAS underground cavern was completed in 2007 and commissioning with cosmic-rays started shortly after completion. During the installation process, the performance of different elements was assessed both on the surface and in the cavern. The first LHC proton-proton collisions took place in December 2009 at a center-of-mass energy of $900 \mathrm{GeV}$ and the SCT was ready to record the delivered collision data. The LHC center-of-mass energy was increased to $7 \mathrm{TeV}$ in March 2010 and since then the SCT is fully powered and delivering good quality data.

At start up, more than $99 \%$ of the 6.3 million readout channels were operational; this percentage has remained stable ever since. Currently there are 30 modules non-operational due to the following reasons: a leaking cooling loop in one of the endcaps (leads to thirteen missing modules), other thirteen modules have powering problems, and few modules have readout problems caused by unexpected failures of off-detector optical transmitters ('TX' channels) that is explained below. All the non-operational channels except for the uncooled modules are distributed randomly over the SCT, so they do not represent a significant loss of coverage in any specific region.

The only serious readout problem experienced by the SCT has to do with the optical laser transmitters, which consist of small VCSEL array cards that plug into the off-detector readout drivers. In late 2008 and early 2009, 'TX' channels were lost at an unacceptable rate as the redundancy scheme does not allow recovery of two adjacent modules with ' $T X$ ' problems. This was traced back to two problems: electrostatic discharge damage during the manufacturing process, which drastically shortened their lifetime, and an unacceptably high sensitivity to humidity. The effects of these problems were minimised by using redundancy wherever possible, replacing the plugins with spares where redundancy was not feasible, and flushing the crates with dry air. However, a long-term solution was needed. New units have been manufactured, which were handled much more carefully with respect to static discharge and are more resistant to humidity. At the time of writing, the plugins are being replaced. Completion is expected by the end of 2011 .

The evaporating cooling system performance is smooth and efficient (except for the aforementioned leaking loop). The SCT operates at $-7^{\circ} \mathrm{C}$ at the silicon surface in the endcaps and $-2^{\circ} C$ in the barrel, except for the outermost barrel layer which operates at $4.5^{\circ} \mathrm{C}$. The barrel operates at higher temperatures in order to compensate for some failed thermal enclosure heaters, ensuring the thermal neutrality of the SCT compared to the Transition Radiation Tracker.

\section{Performance of the SemiConductor Tracker}

In the following, various aspects of the SCT operation demonstrating the performance during 2010 and 2011 collision data taking are described.

\section{A. Noise level}

The noise of the silicon strip modules depends on the detector capacitance, the leakage current as well as on the front-end electronics settings and the operational temperature. Noise can cause a strip to register a hit not caused by the passing of a charged particle through the silicon wafer. The noise occupancy at a given charge threshold is defined as the number of noise hits per channel per nominal LHC bunch 

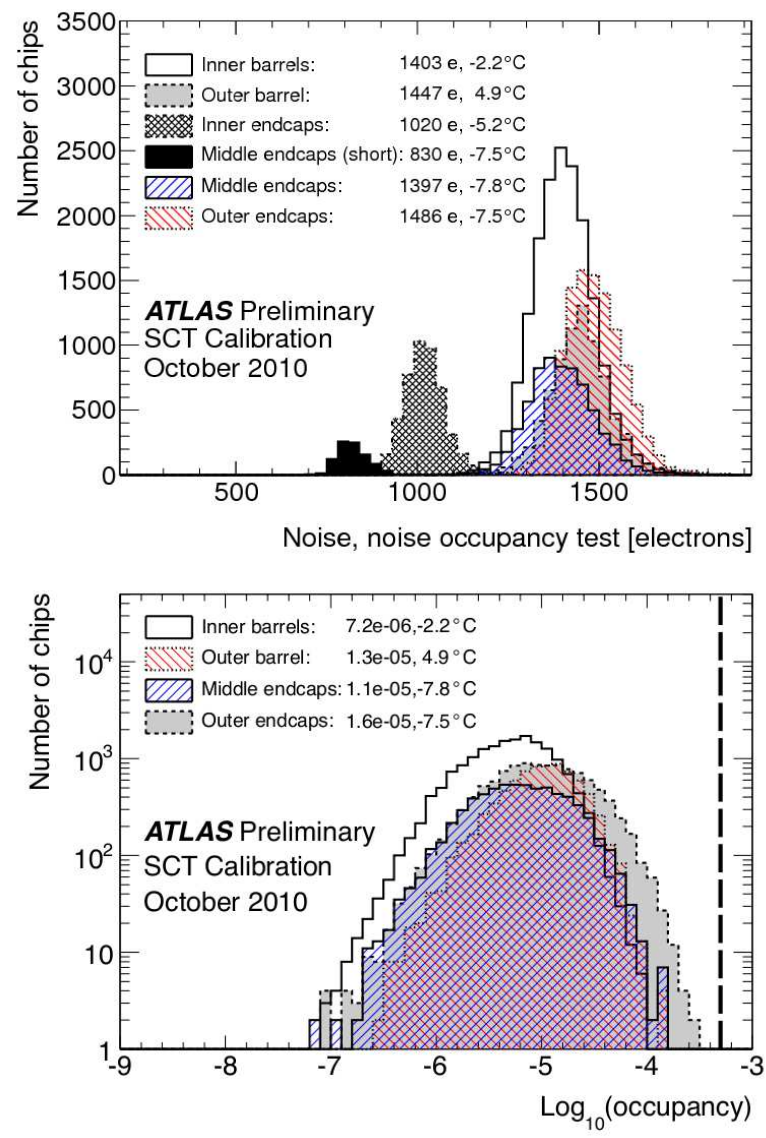

Fig. 4. Top: Distribution of the input noise values for each chip as obtained in response curve measurements. The average values are given (one per region) together with the estimated average silicon temperature during the measurement. Bottom: Noise occupancy averaged over chips in barrel and endcap detectors. The dashed line represents the design requirement. The values for chips on the inner and middle short endcap modules are not shown since in those the average noise lies below the measurement sensitivity. Average values for each detector region are given together with the estimated average silicon temperature.

spacing $(25 n s)$. The design target is $5 \times 10^{-4}$ for a threshold setting of $1 f C$.

The noise occupancy of the SCT detector is measured using two methods: response curve test (in standalone calibration mode) and noise occupancy test (in physics mode running). In the first case, the occupancy at different thresholds is measured by injecting various known charges. For each value of injected charge the threshold is scanned. A response curve is obtained which can be fit to extract a measurement of the input noise. In the second method, the noise level is determined by measuring the noise occupancy as a function of the threshold using random triggers in empty bunch crossings. Results of both methods are shown in Fig. 4 for different types of modules. The two methods give consistent results as can be seen in Fig. 5. The measured noise is well below design specifications for all module types. The different strip lengths and pitch variations are responsible for slightly different occupancy averages for the various module types.

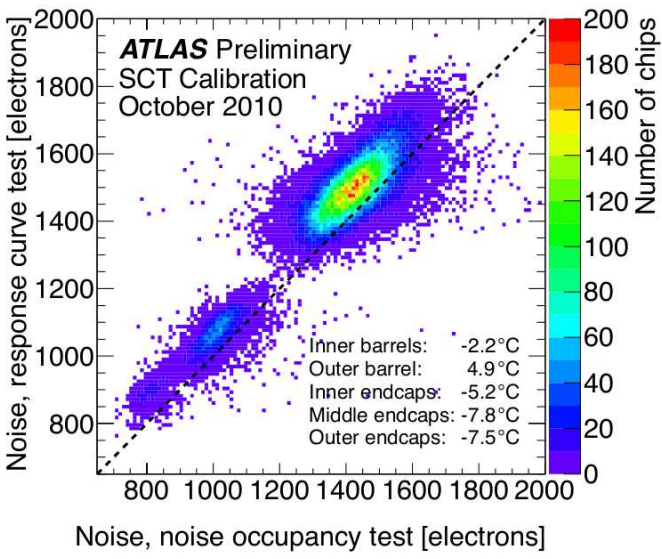

Fig. 5. Comparison of noise occupancy using the two measurements techniques described in the text.

\section{B. Lorentz angle}

As charge carriers drift through the silicon wafer towards the readout strips, they will be deflected by the presence of a magnetic field. The angular deflection relative to the electric field direction is the Lorentz angle, and it depends on the solenoidal field strength, the bias voltage and the temperature of the modules. It is important to monitor the SCT operation conditions and take these into account in the track reconstruction.

The measurement of the Lorentz angle is based on the relationship between the cluster size (number of contiguous hit strips) and the incidence angle of a charged particle track with respect to the sensor. In the absence of a magnetic field, the cluster size would be minimal for particles perpendicular to the sensor. In contrast, with the presence of a magnetic field, the average cluster width is minimal when the charge carriers drift along the direction of the particle track, i.e. the incidence angle of the track is equal to the Lorentz angle. Fig. 6 shows the SCT cluster width (in strips) as a function of the track incidence angle for the barrel layers. The measured Lorentz angles with associated systematic errors and model predictions are shown in Fig. 7. They are consistent. The angles for the outermost layer 3 are different since it is operated at slightly higher temperature.

\section{Timing}

It is crucial to get the timing right relative to the LHC in order to ensure that the data from the correct bunch crossing is read out at the correct time. LHC time bins are 25 ns wide. The SCT reads out three time bins per event, centered around the estimated trigger time. Ideally, a hit should lie in the middle bin, but to be safe, a strip is read out if there was a hit in any of the three bins. This is known as the ' $\mathrm{XXX}$ ' readout mode. 


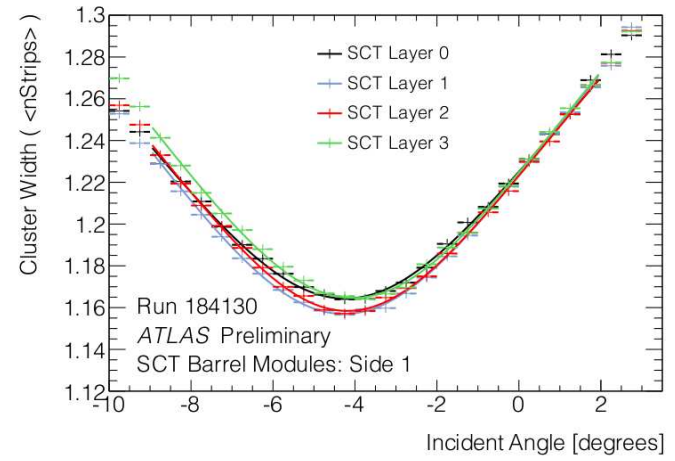

Fig. 6. Average cluster width versus incidence angle of the track with respect to the normal of the surface. The Lorentz angle is given by the minimum of this distribution.

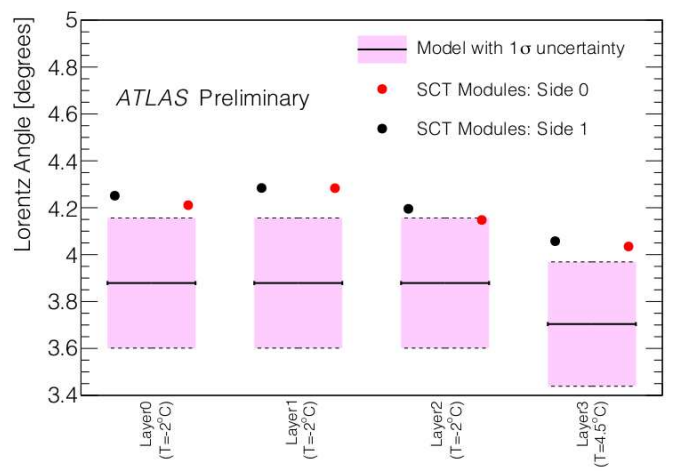

Fig. 7. The values of the extracted Lorentz angle in each layer of the SCT barrel. The measured data values are shown by points for the two module sides. The model prediction ( $1 \sigma$ uncertainty band) that is also shown takes into account the fact that the layers are not operated at the same temperature. The measured values lie within about $1 \sigma$ of the model predictions.

In order to ensure that hits are arriving in the correct time bin, timing scans are performed and adjustments are made in the readout to compensate for differences in the length of the optical fibres and the time-of-flight from the interaction point to the module. Fig. 8 shows the mean time bin of hits in the whole SCT. A value of exactly unity means a pure '010' pattern, i.e. the hit lies on the middle bin. This plot shows that '010' is dominant and therefore that the SCT is well synchronized.

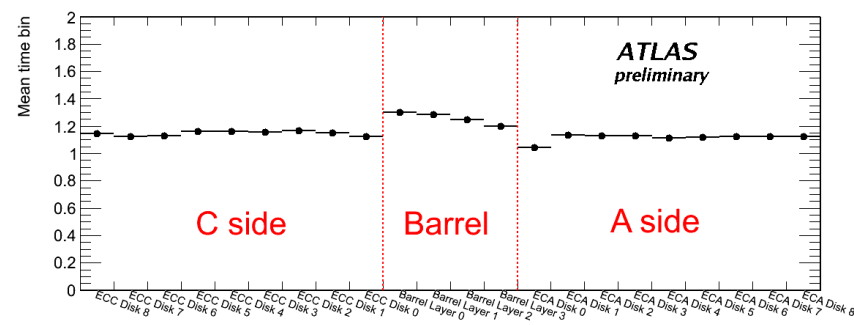

Fig. 8. Mean arrival time bin in the different layers of the SCT.

\section{Intrinsic hit efficiency}

The intrinsic hit efficiency is the number of hits on well-reconstructed tracks divided by the number of possible hits in data. In order to measure it, a 'holes-on-tracks' method is used. The efficiency is computed by counting the number of missing hits (not including the hit under test) in well-reconstructed tracks: $\mathrm{p}_{\mathrm{T}} \geq 1 \mathrm{GeV}$ and at least 7 (6) hits for SCT standalone (Inner Detector combined) tracks. Dead modules and chips are excluded from the efficiency calculation.

Fig. 9 shows the average hit efficiency in each barrel layer and in each disk of one of the endcaps. The 'inner' and 'outer' corresponds to each module side. It includes two types of tracks: SCT standalone and Inner Detector combined tracks. The efficiencies in the innermost (layer 0, inner) and outermost (layer 3, outer) layers are biased in SCT standalone tracks since holes beyond the last measurement are not counted.

The overall hit efficiency is found to be $\sim 99.8 \%$ which is above the design specification.
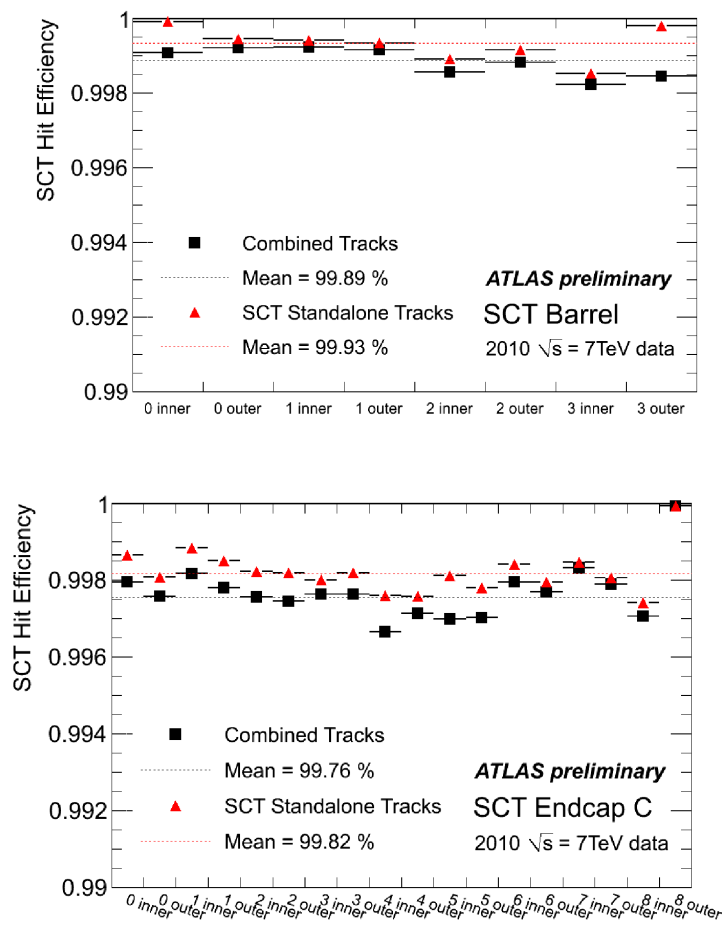

Fig. 9. Average hit efficiency from SCT standalone tracks (red triangles) and combined Inner Detector tracks (black squares) for each barrel layer (top) and disks of one of the endcaps (bottom).

\section{E. Radiation damage}

The collisions at the LHC give rise to radiation levels in the Inner Detector that cause damage to the silicon sensors and electronics. The damage can be monitored by means of the leakage current, which is shown in Fig. 10 for the four 


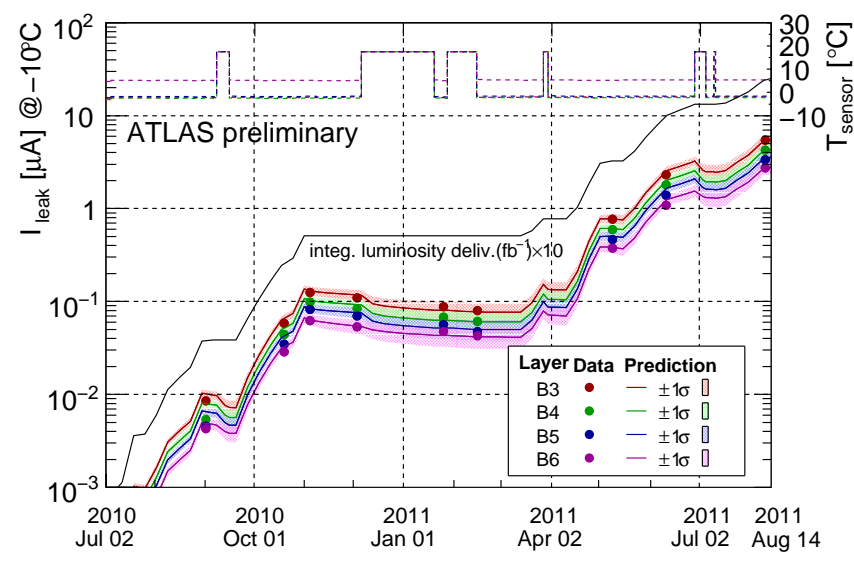

Fig. 10. The leakage current in the SCT barrel layers as a function of time The comparison between measurements and FLUKA predictions shows good agreement.

SCT barrel layers as a function of time. Note that in this plot the nomenclature used is different to that in previous plots, B3 corresponds to layer 0 and so on. The measured values (points) and the predicted ones (shaded area) agree quite well. The predictions come from models of radiation damage in silicon and take into account the luminosity, temperature profile and fluence from FLUKA [10] simulations. Time dependent self-annealing effects, e.g. during winter shutdown period, are taken into account.

\section{Conclusions}

The ATLAS SCT is running smoothly and successfully since its installation. The detector is performing excellently, achieving and surpassing the design requirements. A total of $99.3 \%$ of the SCT modules are operational. The noise occupancy is well below the design requirement of $<5 \times 10^{-4}$. Also, the average hit efficiency exceeds its $99 \%$ design specifications. The optical transmitter failures do not impair data taking efficiency. The new ' $\mathrm{TX}$ ' production is expected to be completed by the end of this year with the full replacement to follow. The detector is well timed-in with the LHC and the trigger system. The sensor behaviour in the $2 T$ solenoidal magnetic field has been studied by measuring the Lorentz angle, which is in good agreement with expectations. The radiation damage is being monitored by periodic measurements of the leakage current and follows nicely the predictions taking operational conditions into account.

\section{REFERENCES}

[1] ATLAS collaboration (A. Abdesselam et al.), The ATLAS Experiment at the CERN Large Hadron Collider, JINST 3, S08003 (2008).

[2] L. Evans and P. Bryant, The LHC machine, JINST 3 S08001 (2008).

[3] ATLAS Inner Detector Technical Design Report, CERN/LHCC97-16 (1997), ISBN 92-9083-102-2.

[4] ATLAS collaboration (A. Abdesselam et al.), The barrel modules of the ATLAS semiconductor tracker, Nucl.Instrum.Meth. A568, p. 642-671 (2006).

[5] ATLAS collaboration (A. Abdesselam et al.), The ATLAS SemiConductor Tracker endcap module, Nucl.Instrum.Meth. A575, p. 353-389 (2007).
[6] F. Campabadal et al., Design and performance of the ABCD3TA ASIC for readout silicon strip in the ATLAS semiconductor tracker, Nucl.Instrum.Meth. A552, 292-328 (2005).

[7] A. Abdesselam et al., The optical links of the ATLAS SemiConductor Tracker, JINST 2, P09003 (2007).

[8] ATLAS collaboration (A. Abdesselam et al.), The data acquisition and calibration system for the ATLAS SemiConductor Tracker, JINST 3, p. P01003 (2008).

[9] D. Attree et al., The evaporative cooling system for the ATLAS inner detector, JINST 3, P07003 (2008).

[10] A. Ferrari, P. Sala, A. Fasso and J. Ranft, FLUKA: A multi-particle transport code, CERN-2005-10 (2005), INFN/TC-05/11, SLAC-R-773. 